\title{
Role of Ser ${ }^{11}$ in the stabilization of the structure of Ochrobactrum anthropi glutathione transferase
}

\author{
Luca FEDERICI* $\dagger$, Michele MASULLI $\uparrow$, Daniele BONIVENTO $\ddagger$, Adele DI MATTEO $\ddagger$, Stefano GIANNI§, Bartolo FAVALORO* $\dagger$, \\ Carmine DI ILIO* ${ }^{*}$ and Nerino ALLOCATI $\uparrow^{1}$ \\ *Ce.S.I. (Centro Studi sull'Invecchiamento), Fondazione Università di Chieti “G. d'Annunzio", Via dei Vestini 31, 66013 Chieti, Italy, †Dipartimento di Scienze Biomediche, \\ Università di Chieti “G. d'Annunzio", Via dei Vestini 31, 66013 Chieti, Italy, łtDipartimento di Scienze Biochimiche, Università di Roma "La Sapienza”, Piazzale A. Moro 5 , \\ 00185 Rome, Italy, and §lstituto di Biologia e Patologia Molecolari del Consiglio Nazionale delle Ricerche, Università di Roma "La Sapienza", Piazzale A. Moro 5, 00185 Rome, Italy
}

\begin{abstract}
GSTs (glutathione transferases) are a multifunctional group of enzymes, widely distributed and involved in cellular detoxification processes. In the xenobiotic-degrading bacterium Ochrobactrum anthropi, GST is overexpressed in the presence of toxic concentrations of aromatic compounds such as 4-chlorophenol and atrazine. We have determined the crystal structure of the GST from $O$. anthropi (OaGST) in complex with GSH. Like other bacterial GSTs, OaGST belongs to the Beta class and shows a similar binding pocket for GSH. However, in contrast with the structure of Proteus mirabilis GST, GSH is not covalently bound to $\mathrm{Cys}^{10}$, but is present in the thiolate form. In our investigation of the structural basis for GSH stabilization, we have identified a conserved network of hydrogen-bond interactions, mediated by
\end{abstract}

the presence of a structural water molecule that links $\operatorname{Ser}^{11}$ to $\mathrm{Glu}^{198}$. Partial disruption of this network, by mutagenesis of Ser ${ }^{11}$ to alanine, increases the $K_{\mathrm{m}}$ for GSH 15-fold and decreases the catalytic efficiency 4-fold, even though Ser $^{11}$ is not involved in GSH binding. Thermal- and chemical-induced unfolding studies point to a global effect of the mutation on the stability of the protein and to a central role of these residues in zippering the terminal helix of the C-terminal domain to the starting helix of the N-terminal domain.

Key words: bacterial glutathione transferase (bacterial GST), circular dichroism, crystallography, G-site, Ochrobactrum anthropi, unfolding.

\section{INTRODUCTION}

GSTs (glutathione transferases) (EC 2.5.1.18) are a family of multifunctional enzymes involved in cell detoxification from xenobiotic compounds [1-5]. The classical reaction catalysed by GSTs is the conjugation of glutathione (GSH) to hydrophobic electrophilic compounds, which generates products that are more easily expelled from the cell [1-4]. Together with this activity, GSTs also show peroxidase and isomerase activities and are capable of binding several substrates non-catalytically [1-4]. GSTs are divided into at least three major families of proteins, namely cytosolic, mitochondrial and microsomal GSTs [5]. The cytosolic GSTs have been subgrouped into several divergent classes on the basis of sequence identity, substrate specificity and type of reaction catalysed [5,6]. Despite interclass sequence identity being often less than $20 \%$, all cytosolic GSTs are dimeric proteins showing a very conserved fold consisting of two domains: a $\mathrm{N}$ terminal thioredoxin-like domain and C-terminal all-helical domain $[3,6]$. Two binding sites are located at the domain's interface: the G-site that accommodates GSH and the H-site where the hydrophobic electrophiles bind [3,6].

Several GSTs of bacterial origin have been grouped into their own class (known as the Beta class) and are characterized by a relatively poor conjugation activity as compared with mammalian GSTs [7]. They are capable of binding several antibiotics, including tetracycline and rifamycin, show some differences in their dimeric organization as compared with mammalian GSTs and perform glutaredoxin-like activity [8-10]. Beta class GSTs are characterized by the presence of a cysteine residue in the G-site [9]. Notably, GSH forms a mixed disulfide with $\mathrm{Cys}^{10}$ in the structure of GST from Proteus mirabilis (PmGST), whereas it is present in the thiolate form in the structure of GST from Burkholderia xenovorans (BxGST) [10,11]. Cys ${ }^{10}$ has been demonstrated to play a role in the glutaredoxin-like reaction catalysed by PmGST [10], while its mutation to alanine did not perturb the classical conjugation activity of PmGST, in a fashion similar to the replacement of the catalytic tyrosine or serine residue of other GST classes, leaving the structural basis of GSH thiolate stabilization in Beta class GSTs unclear [12].

Ochrobactrum anthropi is a xenobiotic-degrading bacterium that is able to proliferate in the presence of highly toxic compounds, such as phenol, 4-chlorophenol and atrazine, and to use them as a carbon source $[13,14]$. The overexpression of the gene encoding a functional GST (OaGST), mainly localized in the periplasm [15], is achieved by the treatment with such aromatic compounds, pointing to the hypothesis that this enzyme serves in cellular detoxification processes $[14,16]$.

In the present study, we have determined the crystal structure of OaGST in complex with GSH and analysed similarities and differences with other bacterial GSTs. We have identified a network of hydrogen-bond interactions, conserved in all Beta class GSTs, that zippers the terminal helix of the C-terminal domain to the starting helix of the thioredoxin-like domain. In order to characterize the role of this network in the G-site and overall

Abbreviations used: CDNB, 1-chloro-2,4-dinitrobenzene; GdmCl, guanidinium chloride; GST, glutathione transferase; BxGST, Burkholderia xenovorans GST; EcGST, Escherichia coli GST; OaGST, Ochrobactrum anthropi GST; PmGST, Proteus mirabilis GST; LB, Luria-Bertani; rmsd, root mean square deviation.

1 To whom correspondence should be addressed (email n.allocati@dsb.unich.it).

The co-ordinates and structure factors for Ochrobactum anthropi glutathione transferase in complex with GSH were deposited in the Protein Data Bank under accession code 2 NTO. 
protein stabilization, we have partially disrupted it by mutating $\operatorname{Ser}^{11}$ to alanine. Our results demonstrate that $\operatorname{Ser}^{11}$ plays a crucial role in both the stability and the functionality of OaGST.

\section{MATERIAL AND METHODS}

\section{Expression and purification of wild-type and mutant OaGST enzymes}

The recombinant OaGST and S11A mutant enzymes were expressed in Escherichia coli XL1-Blue strains as described previously [17]. Briefly, E. coli cells were grown overnight at $37^{\circ} \mathrm{C}$ in LB (Luria-Bertani) medium [18], diluted 1:10 and grown in fresh LB medium until the $D_{550}$ reached 0.4 . To induce gene transcription, IPTG (isopropyl $\beta$-D-thiogalactoside) (SigmaAldrich) was added to a final concentration of $1 \mathrm{mM}$, and the incubation time was prolonged for a further $16 \mathrm{~h}$ after switching the temperature to $25^{\circ} \mathrm{C}$.

The bacterial cells were collected by centrifugation at $5000 \mathrm{~g}$ for $15 \mathrm{~min}$, washed twice and resuspended in $0.025 \mathrm{M}$ imidazole/ $\mathrm{HCl}, \mathrm{pH} 7.4$ (buffer A), containing $2 \mathrm{mM}$ dithiothreitol and disrupted by sonication in the cold. The particulate material was removed by centrifugation at $105000 \mathrm{~g}$ for $60 \mathrm{~min}$, and the resulting supernatant was subjected to chromatofocusing on a column (internal diameter, $1.0 \mathrm{~cm}$; height, $50 \mathrm{~cm}$ ) containing polybuffer exchanger PBE 94 (Amersham Biosciences) equilibrated with buffer A. The column was eluted with polybuffer 74 (Amersham Biosciences) diluted 1:8, pH 4.0 (flow rate, $35 \mathrm{ml} / \mathrm{h}$; fraction volume, $2 \mathrm{ml}$ ). The peak of activity thus separated was concentrated and dialysed against $10 \mathrm{mM} \mathrm{Tris} / \mathrm{HCl}$, pH 7.5 (buffer B), by ultrafiltration in an Amicon apparatus. Concentrated enzyme was purified further by anion-exchange chromatography using a DEAE column (internal diameter, $1.5 \mathrm{~cm}$; height, $11 \mathrm{~cm}$; Bio-Rad Laboratories) equilibrated with buffer B. The enzyme was eluted with a $100 \mathrm{ml}$ linear gradient of $0-0.6 \mathrm{M} \mathrm{KCl}$ in buffer $\mathrm{B}$ (flow rate, $0.5 \mathrm{ml} / \mathrm{min}$; fraction volume, $1 \mathrm{ml}$ ). The peak containing GST activity was pooled, concentrated, dialysed against $10 \mathrm{mM}$ potassium phosphate buffer (pH 7.0) containing $1 \mathrm{mM}$ EDTA by ultrafiltration and subjected to further analyses. SDS/PAGE in discontinuous slab gel was performed by following the method of Laemmli [19]. Protein concentration was determined by following the method of Bradford [20] with $\gamma$-globulin as standard.

\section{Crystallization and data collection}

Wild-type OaGST was extensively dialysed against water and concentrated up to $5 \mathrm{mg} / \mathrm{ml}$. Before crystallization trials, $10 \mathrm{mM}$ GSH was added to the protein sample. Crystals were obtained by the hanging-drop vapour-diffusion method using the following protocol: $1 \mu \mathrm{l}$ of protein sample was mixed in a cover slip with $1 \mu \mathrm{l}$ of a reservoir solution containing $2.0 \mathrm{M}$ ammonium sulfate, $100 \mathrm{mM}$ Tris/ $\mathrm{HCl}, \mathrm{pH} 7.0$, and $200 \mathrm{mM} \mathrm{Li} \mathrm{SO}_{4}$. The coverslip was sealed on a well containing $1.0 \mathrm{ml}$ of the same reservoir solution and equilibrated at $295 \mathrm{~K}$. Crystals appeared after 3 days and reached a final size of $0.1 \mathrm{~mm} \times 0.1 \mathrm{~mm} \times 0.2 \mathrm{~mm}$. The addition of GSH was found to be indispensable for crystal growth. The same or other crystallization conditions failed to produce crystals of the S11A enzyme.

Best data for wild-type OaGST were collected at the ID14-4 beamline of ESRF (European Synchrotron Radiation Facility) synchrotron source (Grenoble, France) equipped with an Oxford cryostream system set at $100 \mathrm{~K}$. Before freezing, crystals were cryoprotected by pouring them in a solution identical with the mother liquor plus $25 \%$ glycerol. Complete data
Table 1 Summary of crystallographic data and refinement

Numbers in parentheses refer to the highest resolution shell.

\begin{tabular}{|c|c|}
\hline Parameter & Value \\
\hline Beamline & ESRF ID14-4 \\
\hline Wavelength $(\AA)$ & 0.934 \\
\hline Resolution (Â) & $50.6-2.1$ \\
\hline Last shell & $2.18-2.10$ \\
\hline$R_{\text {merge }}$ & $0.070(0.335)^{\star}$ \\
\hline Unique reflections & 13632 \\
\hline Completeness (\%) & $99.9(99.8)$ \\
\hline Multiplicity & 13.21 \\
\hline $\mid / \sigma(I)$ & 11.8 \\
\hline Cell dimensions & $a=b=58.765 \AA ; c=212.323 \AA$ \\
\hline Space group & $P 6_{1} 22$ \\
\hline \multicolumn{2}{|l|}{ Refinement } \\
\hline$R$ (working set) & 0.187 \\
\hline$R_{\text {free }}$ (test set) & 0.232 \\
\hline \multicolumn{2}{|l|}{ Rmsd } \\
\hline Bond lengths $(\AA)$ & 0.010 \\
\hline Bond angles $\left({ }^{\circ}\right)$ & 1.307 \\
\hline \multicolumn{2}{|l|}{ Ramachandran statistics } \\
\hline Residues in most favoured regions (\%) & 94.8 \\
\hline Residues in allowed regions (\%) & 4.7 \\
\hline Residues in non-allowed regions (\%) & 0.6 \\
\hline \multicolumn{2}{|l|}{ Model } \\
\hline Amino acids & 1-201 (one monomer in the asymmetric unit) \\
\hline Glutathione & 1 \\
\hline Water molecules & 122 \\
\hline Sulfates & 1 \\
\hline
\end{tabular}

were collected to a resolution of $2.1 \AA(1 \AA=0.1 \mathrm{~nm})$. Data were processed and scaled using DENZO and SCALEPACK respectively [21]. Crystals belong to the space group $P 6_{1} 22$. Unit cell dimensions together with statistics about the data processing are summarized in Table 1.

\section{Structure solution and refinement}

The structure of OaGST was determined by molecular replacement using as a search model the structure of E. coli GST monomer (PDB code 1A0F), including all side chains, but with the exclusion of the glutathione sulfoxide ligand. The program MOLREP from the CCP4 suite was used [22]. The optimal solution yielded a single monomer in the asymmetric unit, in accordance with Matthews coefficient calculations, and the physiological dimer was correctly generated by crystallographic symmetry. With this asymmetric unit composition, the solvent content of the crystal is $49 \%$. This solution was initially refined using rigid body minimization and simulated annealing as implemented in the program CNS [23]. At this stage, electron-density maps were visually inspected and clear electron density for GSH was observed. Model building and refinement were carried out iteratively using COOT [24], to visualize the maps and adjust the model, and restrained refinement as implemented in REFMAC5 [25]. An area of positive electron density in the difference $F_{\mathrm{o}}-F_{\mathrm{c}}$ map, contoured at $3.0 \sigma$, was found at the dimer interface in exact correspondence with the symmetry axis. Given its shape and the crystallization conditions, this area was interpreted by adding a sulfate ion to the model. The final model was refined to a $R$-factor of $18.7 \%$ and a $R_{\text {free }}$ of $23.2 \%$ and contains all 201 residues of the protein, one sulfate ion and 96 water molecules. The final quality of the model is excellent as judged using PROCHECK [26]. Only Gln ${ }^{65}$ has unfavourable stereochemistry according to Ramachandran calculations: this residue is implicated in GSH 
binding and shows the same stereochemistry in all GSTs determined so far. Notably, Tyr $^{86}$ was found to assume two different conformational states in the monomers forming the physiological dimer. Since the dimer is obtained through crystallographic symmetry, Tyr ${ }^{86}$ was modelled in two configurations with halved occupancies. Statistics relative to the refinement and the quality of the model are shown in Table 1.

Structural superimpositions were performed using the program COOT. Contact maps where calculated using the Contact Map Analysis algorithm as implemented in the SPACE server (http:// ligin.weizmann.ac.il/cma). Figures 1 and 3 were prepared using PyMOL (DeLano Scientific).

\section{Enzyme activity}

GST activity with CDNB (1-chloro-2,4-dinitrobenzene) was assayed at $25^{\circ} \mathrm{C}$ according to the method of Habig and Jakoby [27]. For the enzyme kinetic determinations, either CDNB or GSH was held constant at 1 and $5 \mathrm{mM}$ respectively, while the concentration of the other substrate was varied $(0.1-5 \mathrm{mM}$ for GSH and 0.1-1.6 mM for CDNB). Each initial velocity was measured at least in triplicate. The KaleidaGraph Software package (Synergy Software) was used to estimate the Michaelis constant $\left(K_{\mathrm{m}}\right)$ and $V_{\max }$ values by non-linear regression analysis using the MichaelisMenten equation.

The dependence of $k_{\text {cat }} / K_{\mathrm{m}}$ on $\mathrm{pH}$ was determined by using the following buffers $(0.1 \mathrm{M})$ at the indicated $\mathrm{pH}$ values: Bis-Tris/ $\mathrm{HCl}$, from 5.0 to 7.0 , and Tris/ $\mathrm{HCl}$, from 7.2 to 9.0. The reactions were carried out using saturating GSH $(5 \mathrm{mM})$ and variable CDNB concentrations. The $\mathrm{p} K_{\mathrm{a}}$ values were obtained by fitting the data to the equation:

$\log \left(k_{\text {cat }} / K_{\mathrm{m}}\right)=\log \left\{C /\left(1+\left[\mathrm{H}^{+}\right] / K_{\mathrm{a}}\right)\right\}$

where $C$ is the upper limit of $k_{\text {cat }} / K_{\mathrm{m}}$ at high pH [28].

Measurements of enzyme activity $(0.7 \mu \mathrm{M})$ as a function of temperature were carried out by incubating the samples at each temperature for $15 \mathrm{~min}$. GST activity was determined at the end of the incubation.

\section{Unfolding studies}

To study the dependence of enzyme activity on $\mathrm{GdmCl}$ (guanidinium chloride) concentration, wild-type and mutant GST (7 $\mu \mathrm{M})$ were first incubated for $30 \mathrm{~min}$ at $25^{\circ} \mathrm{C}$ in $0.1 \mathrm{M}$ potassium phosphate buffer ( $\mathrm{pH} 6.5$ ) containing $1 \mathrm{mM}$ EDTA with $0-4 \mathrm{M}$ $\mathrm{GdmCl}$. At the end of incubation, each sample was assayed for remaining GST activity in a $1 \mathrm{ml}$ final volume, but including the same concentration of $\mathrm{GdmCl}$ as used in the incubation.

Equilibrium thermal denaturations were followed on a Jasco CD spectrophotometer using a $0.1-\mathrm{cm}$-pathlength quartz cuvette (Hellma). Protein concentration was $5 \mu \mathrm{M}$. Data for wild-type and S11A enzymes were fitted using the equation:

$y_{\mathrm{obs}}=y_{\mathrm{N}}+y_{\mathrm{D}} \frac{e^{a}}{1+e^{a}}$

with

$a=$

$\frac{-\Delta H_{T_{\mathrm{m}}}-\left(1-\frac{T}{T_{\mathrm{m}}}\right)-\Delta c_{\mathrm{p}}\left(T_{\mathrm{m}}-T\right)+\Delta c_{\mathrm{p}}\left[R T \cdot \log \left(\frac{T}{T_{\mathrm{m}}}\right)\right]}{R T}$

where $y_{\mathrm{N}}$ and $y_{\mathrm{D}}$ represent the CD signals of the native and denatured state respectively, $T_{\mathrm{m}}$ is the melting temperature, $\Delta H_{\mathrm{T}_{\mathrm{m}}}$ is the change in enthalpy upon denaturation at the melting temperature and $\Delta c_{\mathrm{p}}$ is the heat capacity change upon unfolding.
In the analysis, the S11A enzyme data were excluded at a temperature of $328 \mathrm{~K}$, because at this temperature the protein grossly precipitates.

GdmCl-induced equilibrium denaturations were performed at $298 \mathrm{~K}$, recording $\mathrm{CD}$ spectra in the interval $215-230 \mathrm{~nm}$. $\mathrm{GdmCl}$ concentration was progressively increased by $0.2 \mathrm{M}$, from 0 to $4.6 \mathrm{M}$, and the spectra were recorded using a $1.0-\mathrm{cm}-$ pathlength quartz cuvette (Hellma) and a protein concentration of $1 \mu \mathrm{M}$. Assuming a standard two-state model, the $\mathrm{GdmCl}$-induced denaturation transition for the wild-type enzyme was fitted to the equation:

$\Delta G_{\mathrm{d}}=\Delta G^{0}-m_{\mathrm{D}-\mathrm{N}} D$

where $\Delta G^{0}$ is the free energy of folding in water and $\Delta G_{\mathrm{d}}$ at a concentration $D$ of denaturant, $m_{\mathrm{D}-\mathrm{N}}$ is the slope of the transition (proportional to the increase in solvent-accessible surface area on going from the native to the denatured state). An equation that takes into account the pre- and post-transition baselines was used to fit the observed unfolding transition [29].

\section{RESULTS AND DISCUSSION}

\section{Overall structure and comparison with Beta class GSTs}

The structure of OaGST was determined by molecular replacement using the structure of $E$. coli GST (EcGST) as a search model [30]. One monomer of OaGST is found in the asymmetric unit, and the physiological dimer is obtained through crystallographic symmetry (Figure 1A). Statistics about data collection and refinement are listed in Table 1.

OaGST displays the typical overall fold of this superfamily of enzymes and is composed of two domains: an N-terminal thioredoxin-like domain (residues $1-76$ ) and a C-terminal $\alpha$-helical domain (residues 89-201), connected by a linker region. Like other bacterial GSTs, OaGST may be classified as belonging to the Beta class. A structure-based comparison with the other three GSTs of known structure belonging to this class, PmGST, EcGST and the recently solved BxGST [11], reveals relatively high sequence identities, i.e. 34,39 and $46 \%$ respectively. This is reflected in the rmsds (root mean square deviations) of equivalent $\mathrm{C} \alpha$ s which are $1.488 \AA$ for OaGST-PmGST, $1.38 \AA$ for OaGSTEcGST and $1.33 \AA$ for OaGST-BxGST. These deviations are smaller than those obtained by comparing OaGST with GSTs representative of other classes. In this case rmsds range from $1.85 \AA$ for the superimposition with the Delta class GST from Anopheles gambiae to $2.67 \AA$ for the superimposition with the murine Pi class GST. Nevertheless, these numbers highlight that the overall GST fold is remarkably well conserved from bacteria to mammals, despite very low interclass sequence identities.

A peculiarity of Beta class GSTs is that their dimer interface is mainly polar and close-packed and differs from the more open and hydrophobic V-shaped interface observed in other classes. The relative orientation of the monomers in the Beta class GST dimers is very well conserved as observed by superimposing the OaGST dimer with the PmGST, EcGST and BxGST dimers (Figure 1B). Rmsds for these superimpositions are 1.614, 1.418 and $1.54 \AA$ respectively. These values are very close to those obtained by superimposing the monomers alone. Interestingly, such a remarkable conservation in the dimer architecture is achieved through a set of interactions that are only partially conserved. This is shown in Figure 2, where interchain contact maps for Beta class GSTs are represented. For instance, a number of contacts between residues belonging to the $\mathrm{C}$-terminal domain of both monomers in PmGST and EcGST (lower right in the contact maps) are not conserved in OaGST and are partially conserved in BxGST. Conversely, in 

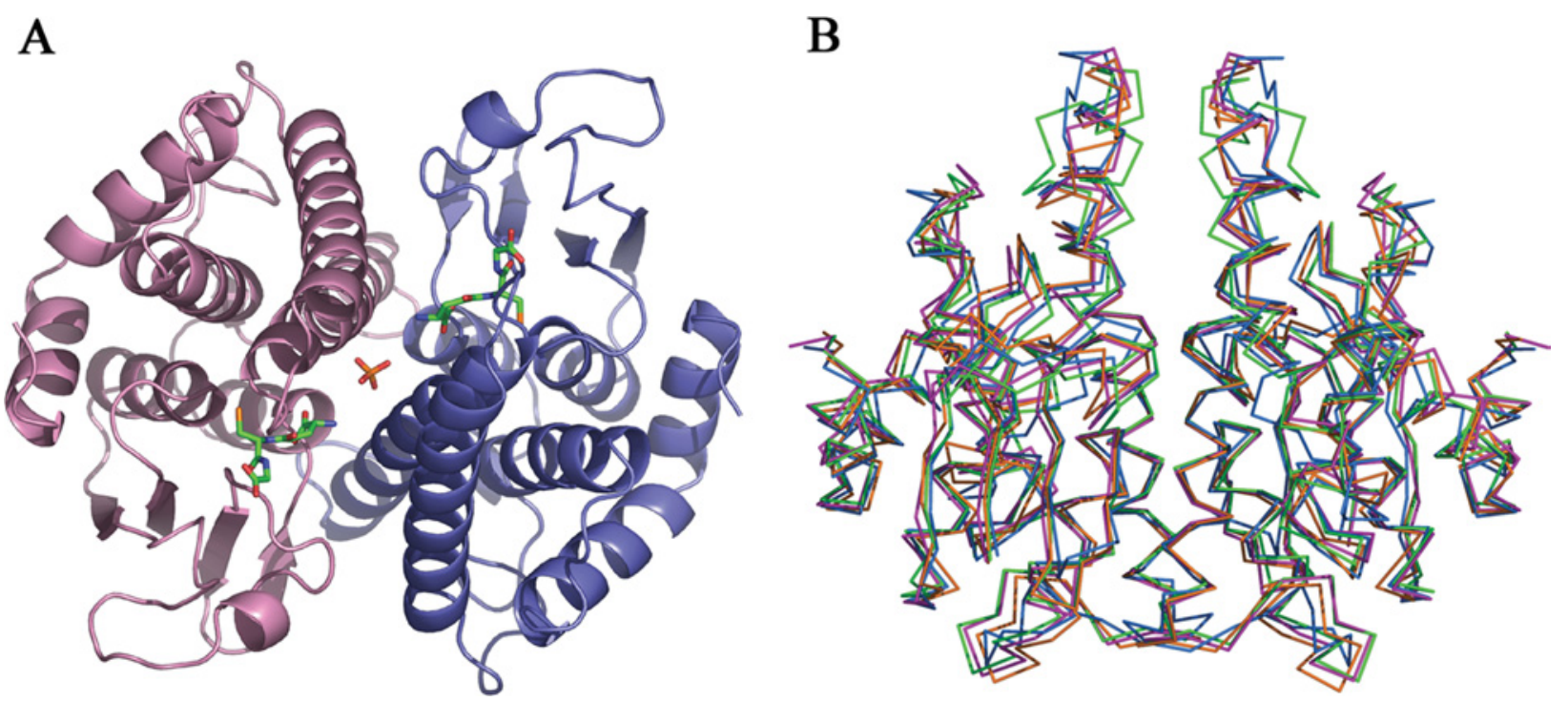

Figure 1 OaGST structure and comparison with GST dimers

(A) Ribbon representation of the physiological dimer of OaGST. Bound GSH molecules are shown as sticks. A sulfate ion, found at the interface between monomers, exactly marks the position of the crystallographic 2-fold axis. (B) Structural superimposition of GST dimers belonging to the Beta class: OaGST (light blue), PmGST (magenta), EcGST (orange) and BxGST (green).

OaGST, a number of contacts between the N-terminal domain of one monomer and the C-terminal domain of the other monomer are not conserved in the other structures (lower left or upper right in the contact maps). Many other differences are also visible throughout the contact maps that provide a fingerprint for each dimer interface. This analysis suggests that, after divergence among the different bacteria, evolution was oriented towards the conservation of the relative orientation of the monomers in the dimers rather than to preserve the chemical nature of the interface.

\section{The G-site}

In the structure of OaGST, GSH is stabilized through a number of ionic and hydrogen-bond interactions with several residues, including $\mathrm{Lys}^{35}$ and $\mathrm{Val}^{52}$, through its peptide carbonyl and amide groups, Asn ${ }^{66}$ and Gln ${ }^{65}$. Similarly to other Beta class GSTs, GSH bound to a monomer is hydrogen-bonded to a residue $\left(\mathrm{Asp}^{103}\right)$ of the other monomer and contributes to the stabilization of the dimer interface in the ligand-bound form. An interesting difference from PmGST is that, in the structure of OaGST, we do not observe a mixed-disulfide bond between GSH and $\mathrm{Cys}^{10}$ (Figure 3). This is also true for the structure of BxGST co-crystallized with GSH [11], while the structure of EcGST was obtained in the presence of the GSH analogue, glutathione sulfonate [30]. In OaGST, the distance between the GSH and enzyme Cys ${ }^{10}$ cysteinyl groups is $3.11 \AA$, suggesting, together with the shape of the electron density, that the GSH is present in the thiolate form and shares a proton with $\mathrm{Cys}^{10}$. The contribution of G-site residues to the thiolate stabilization has been investigated in PmGST and partly in EcGST. In the PmGST structure, three residues were found to be at hydrogen-bond distance from the GSH sulfur, namely $\mathrm{Cys}^{10}, \mathrm{Ser}^{9}$ and $\mathrm{His}^{106}$ (in PmGST numbering). Mutation of Cys ${ }^{10}$ to alanine in both PmGST and EcGST did not affect the conjugating activities of these enzymes, but caused an increase in the GSH pK $K_{\mathrm{a}}[10,31]$. Mutation of Ser ${ }^{9}$ to alanine in PmGST caused a moderate loss in specific activity, but no variation in the $\mathrm{p} K_{\mathrm{a}}$ [12]. Finally, mutation of His ${ }^{106}$ to alanine in PmGST caused a dramatic effect in the catalytic activity, but only a moderate shift to the GSH $\mathrm{p} K_{\mathrm{a}}$ (from 6.4 to 6.7) [32]. By observing the G-site conformation, we note that, in OaGST, as well as in BxGST, the topological position corresponding to the PmGST Ser $^{9}$ is occupied by an alanine residue that cannot contribute to the thiolate stabilization. Moreover, in OaGST, His ${ }^{105}$ occupies a different position from its topologically equivalent His ${ }^{106}$ of PmGST, BxGST and EcGST. This is due to the absence of charge repulsion with the following Lys $^{107}$ that is topologically replaced by an alanine residue in OaGST. As a consequence, OaGST His ${ }^{105} \mathrm{~N} \varepsilon 1$ and $\mathrm{N} \varepsilon 2$ sidechain nitrogens are at a distance of 4.58 and $4.26 \AA$ respectively from the GSH sulfur, as compared with an average distance of $\sim 3.3 \AA$ in the other GSTs, and none of them has a favourable geometry to interact with the GSH sulfur. Thus, in the OaGST structure, two possible contributors to GSH thiolate stabilization are either absent or in an unfavourable position. Nevertheless, the thiolate stabilization achieved in OaGST is more effective than in PmGST, since the $\mathrm{p} K_{\mathrm{a}}$ value for GSH in OaGST is 5.96 (Table 2), whereas in PmGST it is 6.4 [10]. Thus it seems plausible to hypothesize that, in OaGST, Cys ${ }^{10}$ plays an important role both in the stabilization of the GSH thiolate and in defining the conjugation activity.

$\mathrm{Cys}^{10}$ was also found to be crucial for the glutaredoxin-like activity of PmGST [10]. This enzyme is always recovered in the oxidized state after purification, with $\mathrm{Cys}^{10}$ covalently linked to GSH in a mixed disulfide [9]. Interestingly, this is not the case for both OaGST and BxGST that are purified and crystallized in the reduced state. As we have noted above, the main difference at the G-site between PmGST and OaGST/BxGST is the presence in the former of a serine at position 9, establishing a hydrogenbond interaction with the GSH sulfur, that is replaced by an alanine in OaGST, BxGST and EcGST. A possible role of PmGST $\mathrm{Ser}^{9}$ in defining the increased tendency of this enzyme towards the formation of a mixed disulfide as compared with the other Beta class GSTs may thus be hypothesized, but remains to be established.

\section{Mutation of Ser ${ }^{11}$ affects the enzyme activity and G-site stability}

By analysing a sequence alignment of all bacterial GSTs available in the database (results not shown), we observed that, together 


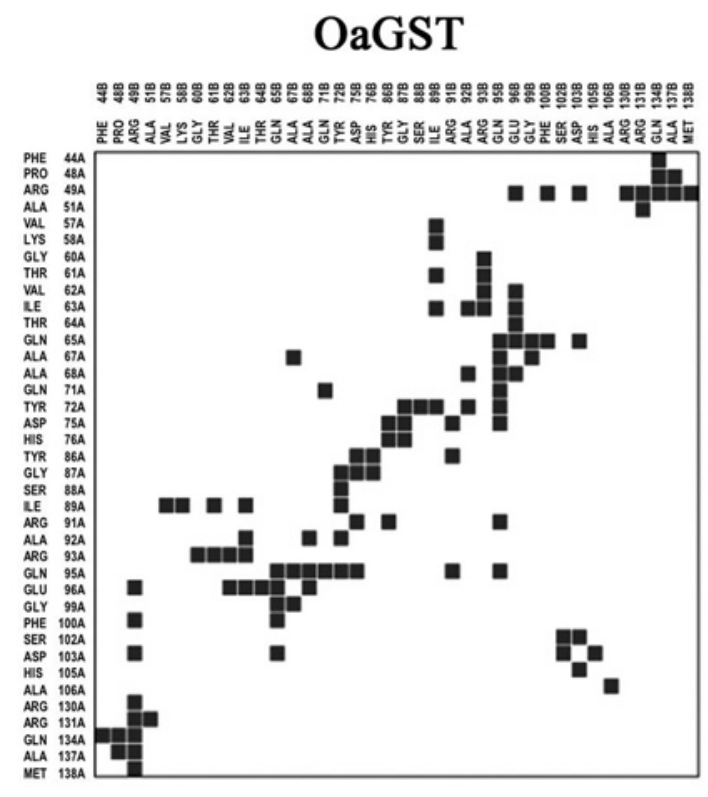

EcGST

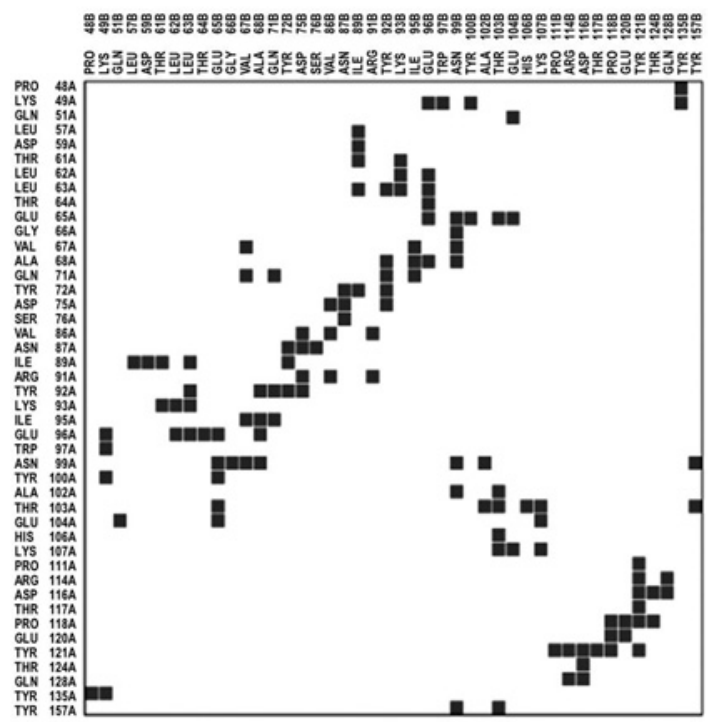

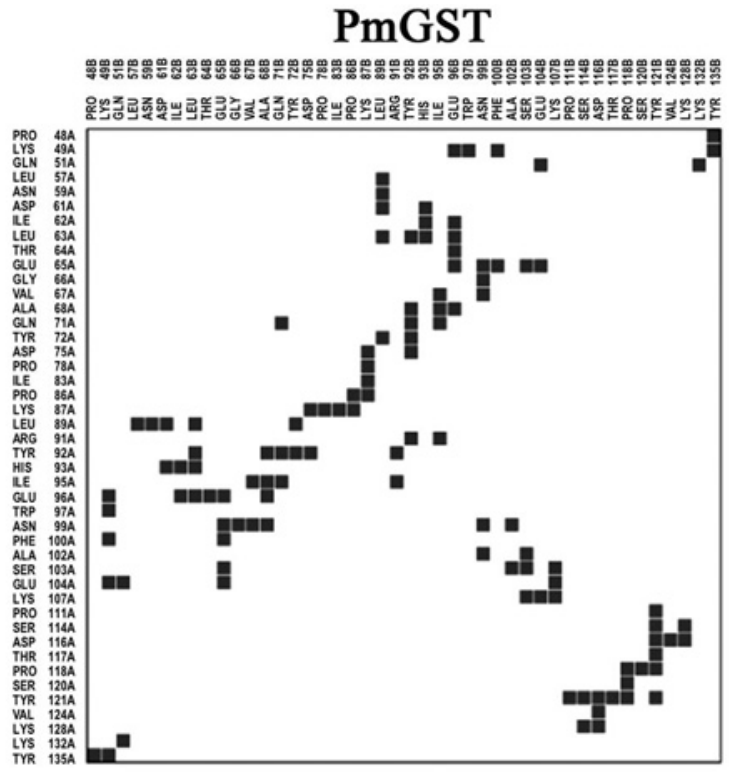

\section{BxGST}

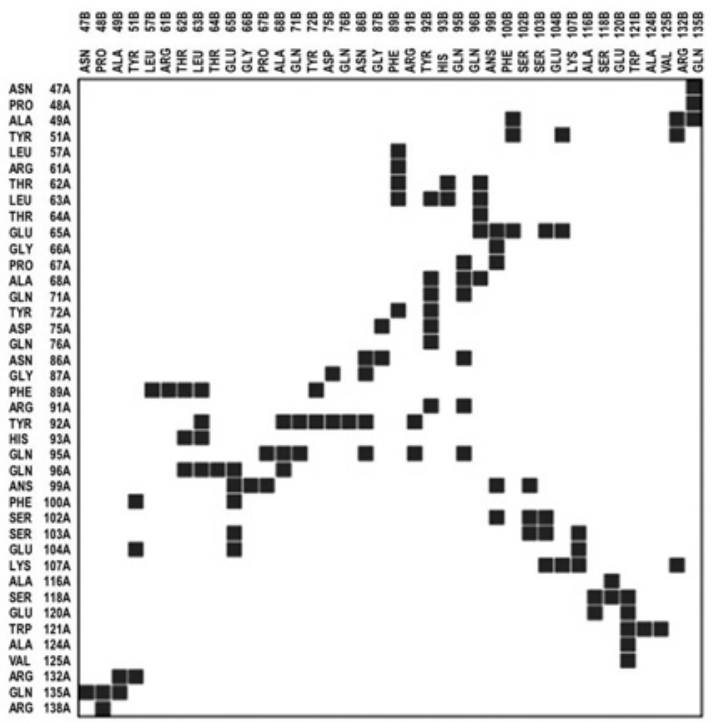

Figure 2 Contact maps for Beta class GSTs

Interactions between residues belonging to different monomers in the dimers are shown as squares. Several differences are observed throughout the maps, even though the orientations of the monomers in the various GST dimers are conserved.

with $\mathrm{Cys}^{10}, \mathrm{Ser}^{11}$ is also completely conserved. Mutation of $\mathrm{Ser}^{11}$ to alanine resulted in a marked loss in activity [17]. The position of this residue in the structure of OaGST attracted our attention, since $\operatorname{Ser}^{11}$ is the first residue of helix 1 and its side chain is not part of the G-site, but points to the opposite direction with respect to $\mathrm{Cys}^{10}$ side chain (Figure 3). The distance between the Ser ${ }^{11}$ hydroxy group and the GSH sulfur is $6.43 \AA$, which excludes a direct role in GSH binding and stabilization. A closer analysis of the structure highlights the presence of a network of hydrogenbond interactions that are conserved in all Beta class GSTs (Figure 3). Three residues take part in this network: Ser $^{11}$ is linked to Glu ${ }^{198}$ through a water molecule that is hydrogen-bonded to both residues. Glu ${ }^{198}$ is in turn also hydrogen-bonded to His ${ }^{15}$. The structural role played by the water molecule is witnessed by its thermal B-factor $\left(23.65 \AA^{2}\right)$ that is in the range of main-chain atom B-factors. The effect of the S11A mutation on enzyme function was tested, and the specific activities and kinetic constants with CDNB as a second substrate for wild-type and S11A OaGST are shown in Table 2. The effect of the mutation is exerted mainly at the G-site. The $K_{\mathrm{m}}$ for GSH is increased considerably (15-fold), while the $k_{\text {cat }} / K_{\mathrm{m}}$ is decreased 5-fold, and the specific activity decreased 3-fold. Interestingly, the $k_{\text {cat }}$ is increased 4-fold. Taken together, these data suggest that the mutation considerably lowers the affinity for GSH and the overall efficiency of the enzyme, but favours the release of the product once the reaction has taken place. Since Ser $^{11}$ does not bind the GSH, this might, in a first instance, be ascribed to an increased mobility and/or destabilization of the G-site in the mutated enzyme. 


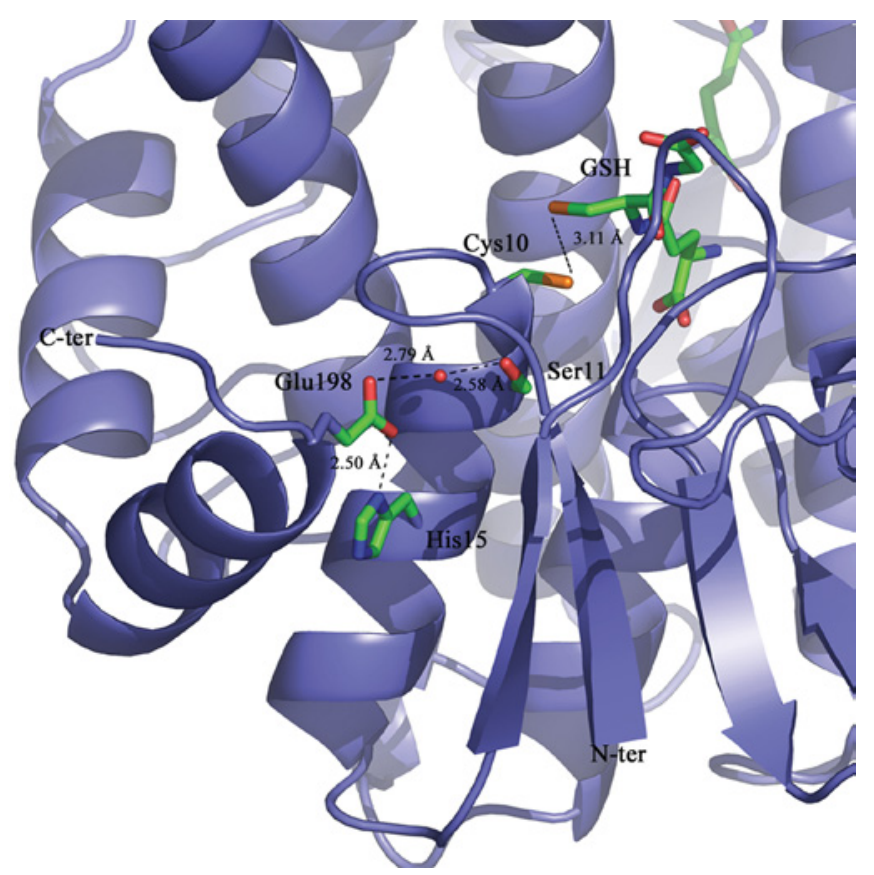

Figure 3 Close-up view of OaGST in the GSH surroundings

Cys $^{10}$ is not covalently linked to the GSH. Ser ${ }^{11}$ is oriented in the opposite direction and interacts with $\mathrm{Glu}^{198}$ via a structural water molecule. Glu ${ }^{198}$ is also hydrogen-bonded to His ${ }^{15}$. These residues are conserved in Beta class GSTs.

The effect on the CDNB kinetic constants is exerted mainly on the $K_{\mathrm{m}}$, but is less evident, in agreement with the observation that this mutation is far from the $\mathrm{H}$-site (Table 2). Interestingly, a long-range effect is also observed in the GSH thiolate stabilization, with a slight decrease of the $\mathrm{p} K_{\mathrm{a}}$ from 5.96 to 5.84 .

To investigate a possible effect on the structural stability of the enzyme G-site, we also followed the conjugation of CDNB by $\mathrm{GSH}$ as a function of temperature and $\mathrm{GdmCl}$ concentration. The thermal stability of the S11A enzyme is compromised: while the wild-type enzyme retains more than $80 \%$ of its functionality at $50{ }^{\circ} \mathrm{C}$, the $\mathrm{S} 11 \mathrm{~A}$ enzyme is already almost inactive at $45^{\circ} \mathrm{C}$ (Figure 4A). Accordingly, the effect of $\mathrm{GdmCl}$ is also markedly different for the S11A enzyme as compared with the wild-type (Figure 4B). At $[\mathrm{GdmCl}]=0.5 \mathrm{M}$, the S11A enzyme completely lost its activity, while the wild-type enzyme still retains $50 \%$ of its functionality.

\section{Global effect of the hydrogen-bond network on OaGST stability}

Next, we wanted to establish whether the effect of the mutation on enzyme kinetics might be ascribed only to a local effect on the G-site conformation, or rather to a global effect on the enzyme stability. Unfortunately, every attempt to crystallize this mutant

\section{A}

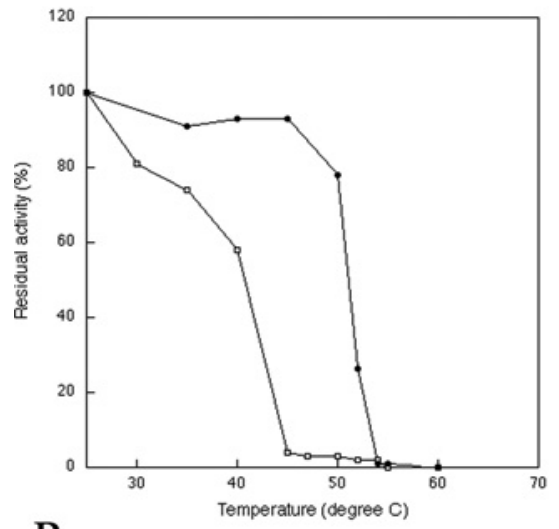

B

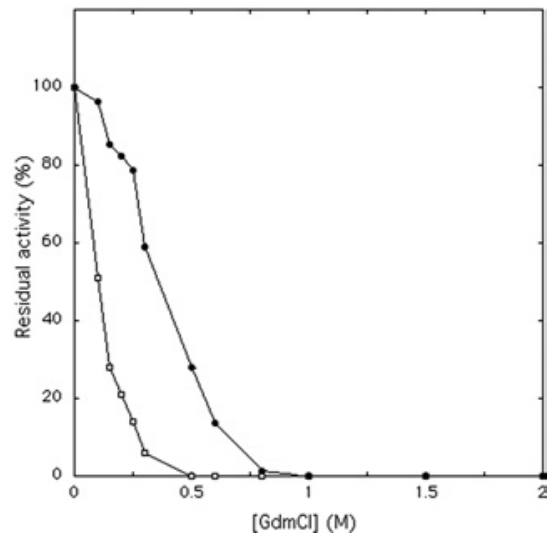

Figure 4 OaGST residual activity with CDNB

(A) OaGST residual activity with CDNB as a function of temperature. (B) OaGST residual activity with $\mathrm{CDNB}$ as a function of increasing concentrations of $\mathrm{GdmCl}$. Wild-type enzyme $(\bullet)$; S11A enzyme ( $\square)$.

failed; however, CD spectra of the wild-type enzyme and the mutated enzyme in the $205-250 \mathrm{~nm}$ interval are completely superimposable (inset in Figure 5), suggesting that the structure of the mutant is native-like under physiological conditions.

Figure 5 shows thermal denaturation of the wild-type and mutated enzymes. Although the wild-type and S11A enzymes display similar native baselines, confirming that the mutated enzyme is fully folded in the physiological temperature range, the transition to the unfolded species is shifted at a lower temperature for the mutated enzyme. This is quantitatively confirmed by fitting the experimental data with a two-state model. Our analysis suggests that the $T_{\mathrm{m}}$ for the mutated enzyme is decreased by $7^{\circ} \mathrm{C}$ (from 56.68 to $49.75^{\circ} \mathrm{C}$ ) and that the overall destabilization caused by the mutation may be estimated as $\Delta \Delta G \sim 1.8 \mathrm{kcal} / \mathrm{mol}(1 \mathrm{kcal} \approx$ $4.184 \mathrm{~kJ})$.

Table 2 Specific activity and kinetic constants for OaGST and the S11A mutant with CDNB as the second substrate

Results are means \pm S.D. for at least three independent determinations.

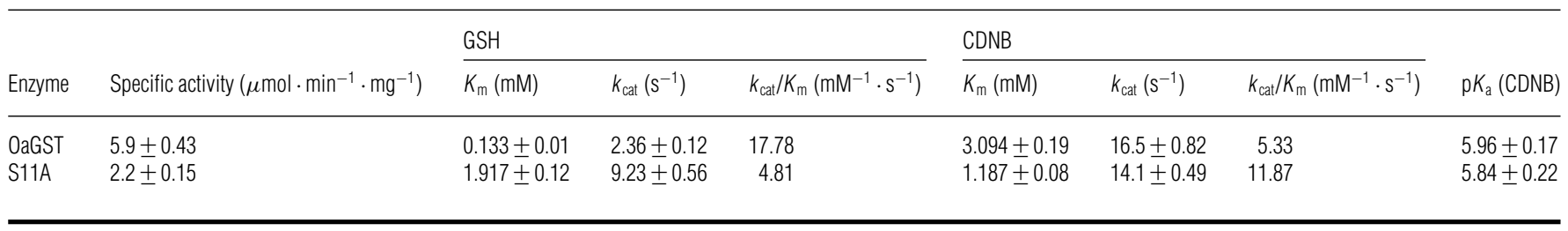




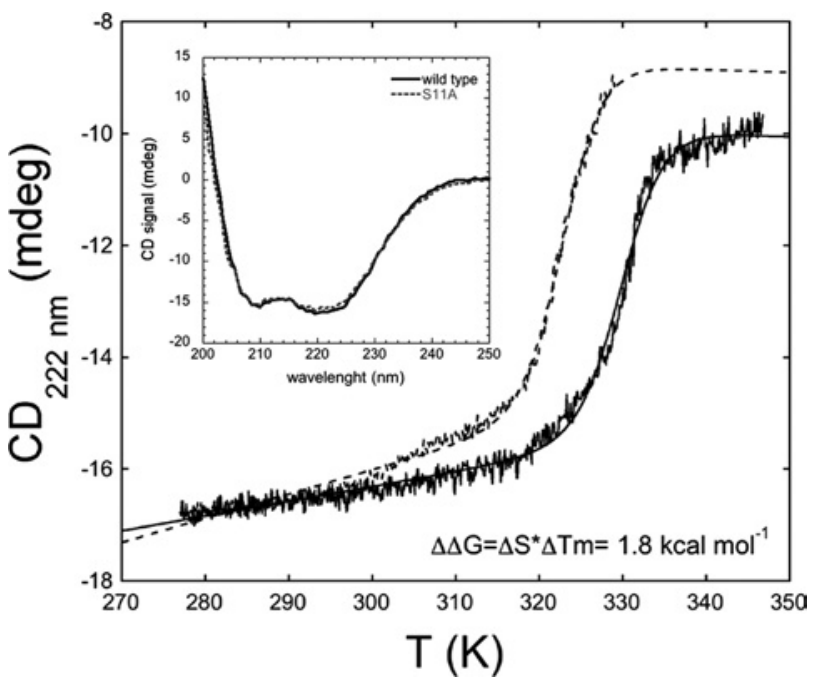

Figure 5 Thermal melting of OaGST measured by CD

Data were fitted to a two-state model. The solid line represents wild-type OaGST data; broken line represents S11A OaGST data. The overall destabilization caused by this single point mutation is estimated to be $1.8 \mathrm{kcal} / \mathrm{mol}$. The inset shows CD spectra for wild-type (solid line) and S11A (broken line) in the interval 200-250 nm.

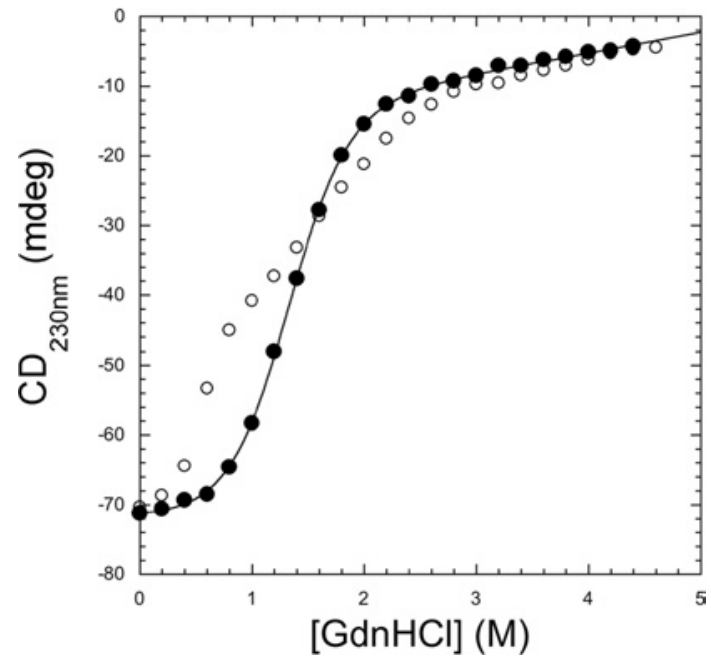

Figure 6 GdmCl-induced equilibrium denaturation of OaGST measured by CD

Wild-type data $(\mathbf{O})$ were fitted to a two-state model. This model could not be applied satisfactorily to the S11A data $(\mathrm{O})$, owing to the appearance of an unfolding intermediate. The overall stability of the protein is estimated to be $2.86 \mathrm{kcal} / \mathrm{mol}$.

We also followed the denaturation of the enzyme in the presence of increasing amounts of $\mathrm{GdmCl}$. In Figure 6, the denaturation profiles for the wild-type and the mutated enzyme are shown, and a different behaviour is observed. Again, a clear transition for the mutated enzyme is visible at very low $\mathrm{GdmCl}$ concentrations where the wild-type enzyme is still fully folded. A closer investigation of the observed denaturation profiles reveals that, while a simple two-state transition can be assigned to the wildtype data, a more complex denaturation is monitored for the S11A mutant. Such behaviour may be consistent with the accumulation of an unfolding intermediate. Taken together, both chemical and thermal unfolding data clearly suggest that a remarkable destabilization is associated with the S11A mutation, i.e. above $60 \%$ of the total free energy of the enzyme, the overall stability of the wild-type protein being approx. $3 \mathrm{kcal} / \mathrm{mol}$.

In present study, we have structurally and functionally characterized the GST from $O$. anthropi, a xenobiotic-degrading bacterium capable of growing in the presence of highly toxic compounds such as 4-chlorophenol and atrazine. On the basis of its structural similarity to previously characterized GSTs, OaGST may be classified as belonging to the Beta class.

Analysing the structure of OaGST, we have identified a previously unrecognized network of hydrogen bonds located at the boundaries of the G-site, but distinct from it, that links the first helix of the $\mathrm{N}$-terminal thioredoxin-like domain to the terminal helix of the $\mathrm{C}$-terminal domain. We have shown that the single mutation S11A dramatically alters the catalytic capabilities of the enzyme towards the model substrate CDNB. This is likely to be the result of the crucial role played by this network in stabilizing the overall fold of the protein, as inferred from chemicaland temperature-induced unfolding studies. Since this hydrogenbond network is conserved in Beta class GSTs, but not in GSTs belonging to other classes, we conclude that, within the framework of the very conserved GST fold, evolution has found different solutions to achieve structural stabilization in the various GST classes.

We thank the beamline scientists at the ESRF (Grenoble, France) for setting up the experimental beamline for $\mathrm{X}$-ray diffraction. This work was partially supported by grants from Ministero dell'Università e della Ricerca.

\section{REFERENCES}

1 Hayes, J. D. and Pulford, D. J. (1995) The glutathione S-transferase supergene family: regulation of GST and the contribution of the isoenzymes to cancer chemoprotection and drug resistance. Crit. Rev. Biochem. Mol. Biol. 30, 445-600

2 Hayes, J. D. and McLellan, L. I. (1999) Glutathione and glutathione-dependent enzymes represent a co-ordinately regulated defence against oxidative stress. Free Radical Res. $\mathbf{3 1}$ 273-300

3 Armstrong, R. N. (1997) Structure, catalytic mechanism, and evolution of the glutathione transferases. Chem. Res. Toxicol. 10, 2-18

4 Mannervik, B. and Danielson, U. H. (1988) Glutathione transferases: structure and catalytic activity. Crit. Rev. Biochem. Mol. Biol. 23, 283-337

5 Hayes, J. D., Flanagan, J. U. and Jowsey, I. R. (2005) Glutathione transferases. Annu. Rev. Pharmacol. Toxicol. 45, 51-88

6 Sheehan, D., Meade, G., Foley, V. M. and Dowd, C. A. (2001) Structure, function and evolution of glutathione transferases: implications for classification of non-mammalian members of an ancient enzyme superfamily. Biochem. J. 360, 1-16

7 Di llio, C., Aceto, A., Piccolomini, R., Allocati, N., Faraone, A., Cellini, L., Ravagnan, G. and Federici, G. (1988) Purification and characterization of three forms of glutathione transferase from Proteus mirabilis. Biochem. J. 255, 971-975

8 Perito, B., Allocati, N., Casalone, E., Masulli, M., Dragani, B., Polsinelli, M., Aceto, A. and Di Ilio, C. (1996) Molecular cloning and overexpression of a glutathione transferase gene from Proteus mirabilis. Biochem. J. 318, 157-162

9 Rossjohn, J., Polekhina, G., Feil, S. C., Allocati, N., Masulli, M., Di llio, C. and Parker, M. W. (1998) A mixed disulfide bond in bacterial glutathione transferase: functional and evolutionary implications. Structure 6, 721-734

10 Caccuri, A. M., Antonini, G., Allocati, N., Di Ilio, C., De Maria, F., Innocenti, F., Parker, M. W., Masulli, M., Lo Bello, M., Turella, P. et al. (2002) GSTB1-1 from Proteus mirabilis a snapshot of an enzyme in the evolutionary pathway from a redox enzyme to a conjugating enzyme. J. Biol. Chem. 277, 18777-18784

11 Tocheva, E. I., Fortin, P. D., Eltis, L. D. and Murphy, M. E. P. (2006) Structures of ternary complexes of BphK, a bacterial glutathione S-transferase that reductively dechlorinates polychlorinated biphenyl metabolites. J. Biol. Chem. 281, 30933-30940

12 Casalone, E., Allocati, N., Ceccarelli, I., Masulli, M., Rossjohn, J., Parker, M. W. and Di llio, C. (1998) Site-directed mutagenesis of the Proteus mirabilis glutathione transferase B1-1 G-site. FEBS Lett. 423, 122-124

13 Laura, D., De Socio, G., Frassanito, R. and Rotilio, D. (1996) Effects of atrazine on Ochrobactrum anthropi membrane fatty acids. Appl. Environ. Microbiol. 62, 2644-2646

14 Favaloro, B., Tamburro, A., Trofino, M. A., Bologna, L., Rotilio, D. and Heipieper, H. J. (2000) Modulation of the glutathione S-transferase in Ochrobactrum anthropi: function of xenobiotic substrates and other forms of stress. Biochem. J. 346, 553-559 
15 Tamburro, A., Robuffo, I., Heipieper, H. J., Allocati, N., Rotilio, D., Di llio, C. and Favaloro, B. (2004) Expression of glutathione S-transferase and peptide methionine sulphoxide reductase in Ochrobactrum anthropi is correlated to the production of reactive oxygen species caused by aromatic substrates. FEMS Microbiol. Lett. 241, 151-156

16 Tamburro, A., Allocati, N., Masulli, M., Rotilio, D., Di llio, C. and Favaloro, B. (2001) Bacterial peptide methionine sulphoxide reductase: co-induction with glutathione S-transferase during chemical stress conditions. Biochem. J. 360, 675-681

17 Favaloro, B., Tamburro, A., Angelucci, S., De Luca, A., Melino, S., Di llio, C. and Rotilio, D. (1998) Molecular cloning, expression and site-directed mutagenesis of glutathione S-transferase from Ochrobactrum anthropi. Biochem. J. 335, 573-579

18 Sambrook, J. and Russell, D. W. (2001) Molecular Cloning: a Laboratory Manual, 3rd edn, Cold Spring Harbor Laboratory Press, Cold Spring Harbor

19 Laemmli, U. K. (1970) Cleavage of structural proteins during the assembly of the head of bacteriophage T4. Nature 227, 680-685

20 Bradford, M. M. (1976) A rapid and sensitive method for the quantitation of microgram quantities of protein utilizing the principle of protein-dye binding. Anal. Biochem. $\mathbf{7 2}$ 248-254

21 Otwinowski, Z. and Minor, W. (1997) Processing of X-ray diffraction data collected in oscillation mode. Methods Enzymol. 276, 307-326

22 Vagin, A. and Teplyakov, A. (2000) An approach to multi-copy search in molecular replacement. Acta Crystallogr. Sect. D Biol. Crystallogr. 56, 1622-1624

23 Brunger, A. T., Adams, P. D., Clore, G. M., DeLano, W. L., Gros, P., Grosse-Kunstleve, R. W., Jiang, J. S., Kuszewski, J., Nilges, M., Pannu, N. S. et al. (1998) Crystallography \& NMR system: a new software suite for macromolecular structure determination. Acta Crystallogr. Sect. D Biol. Crystallogr. 54, 905-921

Received 14 November 2006/2 January 2007; accepted 16 January 2007

Published as BJ Immediate Publication 16 January 2007, doi:10.1042/BJ20061707
24 Emsley, P. and Cowtan, K. (2004) Coot: model-building tools for molecular graphics. Acta Crystallogr. Sect. D Biol. Crystallogr. 60, 2126-2132

25 Murshudov, G. N., Vagin, A. and Dodson, E. J. (1997) Refinement of macromolecular structures by the maximum-likelihood method. Acta Crystallogr. Sect. D Biol. Crystallogr. 53, 240-255

26 Laskowsky, R. A., MacArthur, M. W., Moss, D. S. and Thornton, J. R. (1993) PROCHECK: a program to check the stereochemical quality of protein structures. J. Appl. Crystallogr. 26, 283-291

27 Habig, W. H. and Jakoby, W. B. (1981) Assay for differentiation of glutathione S-transferases. Methods Enzymol. 77, 398-405

28 Liu, S., Zhang, P., Ji, X., Johnson, W. W., Gilliland, G. L. and Armstrong, R. N. (1992) Contribution of tyrosine 6 to the catalytic mechanism of isoenzyme 3-3 of glutathione S-transferase. J. Biol. Chem. 267, 4296-4299

29 Santoro, M. M. and Bolen, D. W. (1988) A test of the linear extrapolation of unfolding free energy changes over an extended denaturant concentration range. Biochemistry $\mathbf{2 7}$, 8063-8068

30 Nishida, M., Harada, S., Noguchi, S., Satow, Y., Inoue, H. and Takahashi, K. (1998) Three-dimensional structure of Escherichia coli glutathione S-transferase complexed with glutathione sulfonate: catalytic roles of $\mathrm{Cys}^{10}$ and His ${ }^{106}$. J. Mol. Biol. 281, $135-147$

31 Inoue, H., Nishida, M. and Takahashi, K. (2000) Effect of Cys ${ }^{10}$ mutation to Ala in glutathione transferase from Escherichia coli. J. Org. Chem. 611, 593-595

32 Allocati, N., Casalone, E., Masulli, M., Polekhina, G., Rossjohn, J., Parker, M. W. and Di llio, C. (2000) Evaluation of the role of two conserved active-site residues in Beta class glutathione S-transferases. Biochem. J. 351, 341-346 